\title{
Actividad acaricida y repelencia de Blechnum cordatum (Blechnaceae) contra Tetranychus urticae (Acari: Tetranychidae)
}

\author{
Acaricidal activity and repellency of Blechnum cordatum (Blechnaceae) against \\ Tetranychus urticae (Acari: Tetranychidae)
}

\author{
CARLOS A. HINCAPIÉ ${ }^{1}$; JULIO ALARCÓN²; ZULMA I. MONSALVE; CARLOS L. CÉSPEDES ${ }^{4}$
}

\begin{abstract}
${ }^{1}$ Doctor en Biología, Grupo de Investigaciones Agroindustriales, Facultad de Ingeniería Agroindustrial, Universidad Pontificia Bolivariana, Sede Central Medellín, Medellín, Colombia, carlos.hincapie@upb.edu.co, https://orcid.org/0000-0002-2652-334X. ${ }^{2}$ Doctor en Química, Laboratorio de Síntesis y Biotransformación, Departamento de Ciencias Básicas, Facultad de Ciencias, Universidad del Bio-Bio, Chillán, Chile, jualarcon@ubiobio.cl, https://orcid. org/0000-0001-7473-8823. ${ }^{3}$ Doctora en Biotecnología de plantas, Grupo de Agrobiotecnología, Instituto de Biología, Universidad de Antioquia, Medellín, Colombia, zmonsalve@gmail.com. https://orcid.org/0000-0002-0583-8086. ${ }^{4}$ Doctor en Química, Laboratorio de Fitoquímica-Ecológica, Departamento de Ciencias Básicas, Facultad de Ciencias, Universidad del Bio-Bio, Chillán, Chile, cespedes.leonardo@gmail.com, https://orcid.org/0000-0002-3715-3268.
\end{abstract}

\begin{abstract}
Autor para correspondencia: Carlos A. Hincapié, Doctor en Biología, Grupo de Investigaciones Agroindustriales, Facultad de Ingeniería Agroindustrial, Universidad Pontificia Bolivariana, Sede Central Medellín, Circular $1^{\text {a }}$ No. 70-01, Medellín, Colombia, carlos.hincapie@upb.edu.co, https://orcid. org/0000-0002-2652-334X.
\end{abstract}

Citación sugerida / Suggested citation: HINCAPIÉ, C. A.; ALARCÓN, J.; MONSALVE, Z. I.; CÉSPEDES, C. L. 2019. Actividad acaricida y repelencia de Blechnum cordatum (Blechnaceae) contra Tetranychus urticae (Acari: Tetranychidae). Revista Colombiana de Entomología 45 (2): e7957. https://doi.org/10.25100/socolen.v45i2.7957

Recibido: 16-feb-2018

Aceptado: 11-mar-2019

Publicado: 16-ene-2020

Revista Colombiana de Entomología

ISSN (Impreso): 0120-0488

ISSN (En línea): 2665-4385

http://revistacolombianaentomologia.univalle.edu.co/

Open access

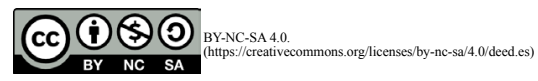

Publicadores / Publishers:

Sociedad Colombiana de Entomología

SOCOLEN (Bogotá, D. C., Colombia)

http://www.socolen.org.co

Universidad del Valle (Cali, Colombia)

http://www.univalle.edu.co/

(C) 2019 Sociedad Colombiana de Entomología - SOCOLEN y Universidad del Valle - Univalle
Resumen: Tetranychus urticae es una especie de ácaro altamente polífago, con registros en 1.140 especies hospederas a nivel mundial. En el presente estudio, se reporta la toxicidad y la repelencia de Blechnum cordatum contra $T$. urticae. Con relación a la toxicidad, las fracciones obtenidas con acetato de etilo a $250 \mathrm{ppm}$ y $n$-hexano a $250 \mathrm{ppm}$ y $100 \mathrm{ppm}$ causaron las más alta tasas de mortalidad acumulada cuando se hizo la aplicación desde el estadio larval. Los resultados del estudio muestran que aún a las más bajas concentraciones evaluadas (10 ppm), ambas fracciones causaron una disminución significativa en la población del ácaro. La fracción hexánica causó $100 \%$ de repelencia a $50 \mathrm{ppm}$ y una repelencia significativamente superior al control a $10 \mathrm{ppm}$. Se encontró una relativa baja actividad cuando los tratamientos se aplicaron directamente a adultos. Los resultados permiten inferir que la mortalidad acumulada pudo haber sido causada por inanición dada la actividad repelente o la muy bien conocida actividad disuasora de los fitoecdisteroides (FEs) presentes en los extractos. Se aislaron e identificaron cuatro FEs a partir de la fracción hexánica de B. cordatum: ecdisona, ponasterona A, shidasterona y 2-deoxicrustecdisona.

Palabras clave: Extractos de plantas, Blechnaceae, control de plagas, fitoecdisteroides, ecdisteroides, Acari, Tetranychidae.

Abstract: Tetranychus urticae is a highly polyphagous mite species, with 1,140 host plant species reported worldwide. In this study, both Blechnum cordatum toxicity and repellent activity against $T$. urticae are reported. Regarding toxicity, the ethyl acetate fraction at 250 ppm and the $n$-hexane fractions at $250 \mathrm{ppm}$ and $100 \mathrm{ppm}$ caused the greatest accumulated mortality rate when application was made starting during the larval stage. Results showed that even at the lowest evaluated concentration $(10 \mathrm{ppm})$, both fractions caused a significant decrease in the $T$. urticae population. The $n$-hexane fraction caused $100 \%$ repellency at 50 $\mathrm{ppm}$ and repellency significantly superior to the control at $10 \mathrm{ppm}$. Relatively low activity was found when treatments were applied directly to adults. In addition, results suggest that the cumulative mortality could have been caused by starvation due to the observed repellency and the well-known deterrent activity of phytoecdysteroids (PEs) found in the extracts. Four PEs from the $B$. cordatum $n$-hexane fraction were isolated: ecdysone, ponasterone $\mathrm{A}$, shidasterone and 2-deoxycrustecdysone.

Keywords: Plant extracts, Blechnaceae, pest control, phytoecdysteroids, ecdysteroids, Acari, Tetranychidae.

\section{Introducción}

La arañita roja bimaculada, Tetranychus urticae (Koch, 1836) (Acari: Tetranychidae), es un ácaro plaga altamente polífago y cosmopolita, con presencia en 1.140 especies de plantas (Migeon y Dorkeld 2017). Algunas poblaciones de este ácaro han desarrollado resistencia a diferentes principios activos utilizados para su control (Van Leeuwen et al. 2010), causando que se deban utilizar mayores cantidades de producto, mayor número de aplicaciones y combinación de diferentes productos comerciales en su control. Esto además conduce a una disminución significativa de la fauna benéfica. La búsqueda de nuevos principios activos y la creciente popularidad de la producción orgánica han motivado numerosas investigaciones en el uso de 
plantas para encontrar alternativas para controlar este ácaro (Hincapié et al. 2008; Afify et al. 2012; Araújo et al. 2012).

Una planta que se ha estudiado, y a la que se le ha encontrado cierto grado de toxicidad, es Blechnum cordatum (Desv.) Hieron, especie común en Centroamérica, Las Antillas y en todos los países de Sudamérica (Prada et al. 2008; Ramos et al. 2006). Se ha determinado in vitro que diferentes extractos de esta planta causan mortalidad sobre Galleria mellonella L., 1756 (Lepidoptera: Pyralidae) (Zapata et al. 2016) y muestran actividad inhibidora del crecimiento en Drosophila melanogaster (Meigen, 1830) (Diptera: Drosophilidae) (Hincapié et al. 2011) y de G. mellonella (Zapata et al. 2016). Uno de los grupos de compuestos que se encuentran en la familia Blechnaceae, a la que pertenece esta especie, son los fitoecdisteroides (FEs), también conocidos como fitoecdisonas (Prada et al. 2008; Hincapié et al. 2011). Los FEs son triterpenos esteroidales con estructura similar a los ecdisteroides que se encuentran de forma natural en insectos. En las plantas participan en la defensa contra invertebrados fitófagos no adaptados, principalmente porque poseen actividad que interfiere con los procesos de muda de los mismos (Gorelick-Feldman et al. 2008) o por su actividad disuasora de la alimentación (Malausa et al. 2006; Rharrabe et al. 2011). Los FEs, junto con otros terpenoides (drimanos, clerodanos, limonoides y agarofuranos), son los más promisorios agentes antialimentarios en plantas, que aunque pueden ser tóxicos, matan principalmente a los insectos de hambre (Descoins 2001). Se ha determinado que los FEs presentes en diferentes especies de helechos son los responsables de que sean relativamente resistentes al ataque de insectos (Mannan et al. 2008). Se ha encontrado que al alimentar larvas de Acrolepiopsis assectella (Zeller, 1839) (Lepidoptera: Acrolepiidae) con dietas que contienen FEs se inducen efectos patofisiológicos (Arnault y Sláma 1986). Las dietas ricas en dichos compuestos causaron una mortalidad del $80 \%$ en el segundo ínstar de la larva de Cryptorhynchus lapathi L., 1758 (Coleoptera: Curculionidae) (De-fu et al. 2002).

Los ecdisteroides son hormonas que juegan un papel esencial en la muda y la metamorfosis de los insectos (Riddiford 2012). Los mecanismos de regulación del desarrollo y la reproducción por parte de estos compuestos han sido investigados exhaustivamente en los órdenes Diptera y Lepidoptera, pero pocos estudios se han realizado para identificarlos y determinar su papel sobre la fisiología de otros insectos y otros artrópodos, tales como los ácaros (Horigane et al. 2007). En estos últimos se destacan unos pocos estudios en garrapatas (orden Ixodida Leach, 1815) enfocados principalmente en vitelogénesis y oogénesis (Horigane et al. 2007; Ogihara et al. 2007; Seixas et al. 2008; Cabrera et al. 2009) y con algunos concentrados en muda (Horigane et al. 2007; Horigane et al. 2008; Seixas et al. 2008). En ellos se concluyó que los ecdisteroides pueden ser las hormonas principales reguladoras del crecimiento en las especies de garrapatas estudiadas (Horigane et al. 2007). Un trabajo realizado sobre Oligonychus perseae (Tuttle, Baker y Abbatiello, 1976) (Acari: Tetranychidae) determinó que los compuestos 20-hidroxiecdisona (20-E) y ciasterona tuvieron una influencia significativa sobre la fecundidad y la mortalidad de adultos (Aly et al. 2011). Se han reportado efectos de los ecdisteroides y FEs en mamíferos, tales como el incremento en la síntesis de proteína de los músculos esqueléticos (GorelickFeldman et al. 2008), efectos anabólicos (Bathori et al. 2008), inhibición de células MCF-7, línea celular del cáncer derivada del adenocarcinoma de pecho (Gaube et al. 2008), corrección de la hiperglicemia y prevención de las complicaciones derivadas de la diabetes en el hígado, páncreas y riñones en ratas (Hamden et al. 2008). El objetivo de este estudio fue evaluar la toxicidad y la repelencia de diferentes fracciones obtenidas a partir de B. cordatum sobre $T$. urticae y aislar e identificar los fitoecdisteroides presentes en las más efectivas.

\section{Materiales y métodos}

Material vegetal. Se recolectaron hojas jóvenes de $B$. cordatum en la ribera sur del río Chillán $\left(36^{\circ} 38^{\prime} 33,47^{\prime} \mathrm{S}\right.$, $72^{\circ} 12$ '40,42”O, $81 \mathrm{msnm}$ ), Provincia del Nuble, Región del Bio-Bio, Chile, en mayo de 2010. Se depositaron los especímenes en el herbario del departamento de Ciencia Básica de la Universidad del Bio-Bio (Voucher No: 2010/05).

Preparación de extractos y fracciones. Muestras del material vegetal de $B$. cordatum recolectadas se deshidrataron usando aire a temperatura ambiente $\left(20 \pm 5{ }^{\circ} \mathrm{C} ; 68 \pm 5 \%\right.$ HR; 72 h.). Enseguida, se sometieron a molienda usando un molino manual. El material resultante $(646,5 \mathrm{~g})$ se sometió a extracción con metanol $1(1: 3, \mathrm{p} / \mathrm{v})$ durante 24 horas, cinco veces. El extracto resultante se concentró a presión reducida a $40{ }^{\circ} \mathrm{C}$ usando un rotoevaporador (Waterbath-B-480, Büchi Labortechnik AG, Flawil, Suiza) para eliminar en su totalidad el metanol. El extracto obtenido (198,2 g) se disolvió en una solución 60/40 metanol/agua destilada $(1: 3, \mathrm{p} / \mathrm{v})$ y se puso en embudo de separación donde se lavó con $n$-hexano (150 $\mathrm{mL}$, cinco veces). La fase hexánica se concentró a presión reducida a $40{ }^{\circ} \mathrm{C}$ usando un rotoevaporador. La fase metanol/ agua resultante se sometió al mismo procedimiento usando acetato de etilo (EtOAc) $(1: 1, \mathrm{v} / \mathrm{v})$. Las dos fases resultantes se concentraron a presión reducida a $40{ }^{\circ} \mathrm{C}$ usando un rotoevaporador (Muñoz et al. 2013).

Cría del ácaro. Se colectaron especímenes de $T$. urticae en diferentes cultivos de la región y se multiplicaron sobre plantas de frijol cargamanto (Phaseolus vulgaris L.) en condiciones de invernadero en el Campus Laureles de la Universidad Pontificia Bolivariana, Medellín, Colombia de acuerdo con el método citado por Hincapié et al. (2008). Las condiciones de la ciudad son $22,5 \pm 5,5^{\circ} \mathrm{C}$, HR $68 \pm 5$ $\%$, fotoperiodo promedio L12:O12 y $1497 \mathrm{msnm}$ ) (IDEAM 2014). Las condiciones ambientales del invernadero no fueron controladas ni medidas.

Biensayo de mortalidad acumulada sobre $T$. urticae. Todos los experimentos con el ácaro se realizaron en condiciones de laboratorio, controladas mediante el sistema de acondicionamiento de aire y sistema de iluminación (temperatura $23 \pm 2{ }^{\circ} \mathrm{C}, \mathrm{HR} 62 \pm 2 \%$, fotoperiodo promedio L12: O12). Se transfirieron y mantuvieron por 48 horas (Miresmailli e Isman 2006) 100 hembras adultas de T. urticae a la cara abaxial de hojas de $P$. vulgaris, de esta forma se obtuvieron huevos de aproximadamente la misma edad. Esta operación se repitió todas las veces que fue necesario durante el experimento. Se recolectaron 20 huevos de forma aleatoria para cada una de las repeticiones. Una vez obtenido el material biológico se prepararon tres concentraciones (10, 100 y $250 \mathrm{ppm}$ ) a partir de las fracciones con acetato de etilo y $n$-hexano disolviéndolas en agua destilada y usando tween 
$80(0,02 \%)$ como emulsificante, además un testigo negativo (agua destilada y la misma concentración de tween 80 ). No se usó testigo positivo. Discos de hoja de $14 \mathrm{~mm}$ de diámetro de $P$. vulgaris fueron sumergidos en cada solución durante diez segundos y se dejaron secar hasta que no presentaran humedad en su superficie. Los discos se ubicaron sobre papel de filtro humedecido para mantener su humedad, con su cara abaxial hacia arriba, en cajas de Petri de cuatro $\mathrm{cm}$ de diámetro. Se ubicaron los huevos recolectados sobre la superficie de cada disco de hoja tratado (Hincapié et al. 2008). Se dejaron eclosionar y se tomó como población inicial las larvas eclosionadas. Se registraron cada 24 horas los individuos vivos y muertos en cada estadio de desarrollo (larva, ninfa y adulto) durante 12 días, cuando se dio por terminado el experimento (adaptado de Hincapié et al. 2011). Se consideraron muertos si no se observaba movimiento después de ser tocados suavemente en varias ocasiones con un pincel. Los valores porcentuales de individuos en cada estadio fueron calculados usando las siguientes fórmulas:

$$
\begin{aligned}
& \begin{array}{c}
\text { Porcentaje de } \\
\text { individuos } \\
\text { muertos por } \\
\text { estadío }
\end{array} \\
& \begin{array}{c}
\text { Porcentaje de } \\
\text { mortalidad } \\
\text { acumulada }
\end{array}
\end{aligned}=\frac{\mathrm{n} \text { muertos en cada estadío }}{\mathrm{n} \text { vivos en cada estadío }} \times 100
$$

donde $\mathrm{n}$ es el número de ácaros. Los resultados se corrigieron usando la fórmula de Abbott (1925).

Bioensayo de mortalidad en adultos de $T$. urticae. Se evaluaron cuatro concentraciones de las fracciones obtenidas con acetato de etilo y $n$-hexano $(250,1.000,2.500$ y 5.000 ppm) de acuerdo con pruebas preliminares. Todas las fracciones fueron disueltas en agua destilada usando tween $80(0,02$ $\%)$ como emulsionante. El testigo negativo se preparó solo con agua destilada y tween $80(0,02 \%)$ y un testigo positivo usando un producto comercial con base en abamectina al 1,8 $\%$ a 750 ppm (dosificación recomendada por el fabricante). Discos de hoja de $30 \mathrm{~mm}$ de diámetro fueron tratados como se ha descrito en los bioensayos anteriores. Se colocaron 15 hembras adultas de T. urticae, seleccionadas al azar de la cría establecida, en la cara abaxial de los discos tratados y se registró la mortalidad a las 24,48 y 72 horas después de iniciado el experimento. La mortalidad se verificó como se describió (Hincapié et al. 2008; Pavela et al. 2016). El porcentaje de mortalidad fue calculado así:

$$
\underset{\text { Porcentaje de }}{\text { mortalidad }}=\frac{\mathrm{n} \text { muertos en to }}{\mathrm{n} \text { vivos en ti }} \times 100
$$

donde $\mathrm{n}$ es el número de ácaros, ti es el tiempo cero o de inicio del experimento, to es el tiempo de observación. Los resultados se corrigieron usando la fórmula de Abbott (1925).

Bioensayo para determinar repelencia sobre $T$. urticae. Se siguió el protocolo establecido por Boyd y Alverson (2000) adaptado por Hincapié et al. (2008). Se evaluaron tres concentraciones de las fracciones obtenidas con acetato de etilo (100, 250 y $500 \mathrm{ppm})$ y con $n$-hexano (1, 10 y $50 \mathrm{ppm})$. Todas las fracciones fueron disueltas en agua destilada usando tween $80(0,02 \%)$ como emulsionante. El testigo negativo se preparó usando solo agua destilada y tween $80(0,02 \%)$ y el testigo positivo usando un producto comercial con base en ajo a 750 ppm, por la comprobada actividad repelente de ese tipo de extractos (Boyd y Alverson 2000; Dąbrowski y Seredyńska 2007; Hincapié et al. 2008). Discos de hoja de $P$. vulgaris de $35 \mathrm{~mm}$ de diámetro fueron sumergidos dentro de cada solución, se dejaron secar hasta que no se observara humedad en su superficie y se ubicaron con la cara abaxial hacia arriba sobre papel filtro humedecido para mantener su humedad en cajas de Petri de $4 \mathrm{~cm}$ de diámetro. Se ubicó en el medio del disco tratado un disco de hoja de $14 \mathrm{~mm}$ de diámetro sin tratar (DST) con su cara abaxial hacia arriba y a ese disco se transfirieron 15 hembras adultas de T. urticae, seleccionadas aleatoriamente de la cría establecida. Se registraron los ácaros que se mantenían en el disco sin tratar 1, 2, 3, 4 y 24 horas. El porcentaje de repelencia fue calculado de la siguiente manera:

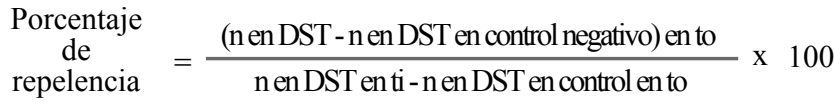

donde $\mathrm{n}$ es el número de ácaros, ti es el tiempo cero o de inicio del experimento, to es el tiempo de observación y DST es el disco sin tratar.

Diseño experimental y análisis estadístico. Se usaron diseños completamente al azar para cada uno de los experimentos (Edwards et al. 2010; Sivira et al. 2011) con cinco repeticiones por tratamiento. Se aplicaron las pruebas de Shapiro-Wilk y Levene para verificar los supuestos de la varianza (normalidad y homocedasticidad, respectivamente). Los resultados porcentuales, en fracción, en cada experimento fueron transformados usando la función arcoseno, $\mathrm{Vx} / 100$, previamente a la ejecución del análisis de varianza (ANOVA) $(\alpha=0,05)$ en cada tiempo de observación. Se usó la prueba de Tukey cuando se encontraron diferencias estadísticamente significativas entre los tratamientos. Se usó el programa SAS 8.0 (SAS Institute Inc. 1999) para mortalidad y repelencia de adultos y el programa Statgraphics ${ }^{\circledR}$ Centurion XVI (Statgraphics Technologies 2013) para mortalidad acumulada y determinación de $\mathrm{CL}_{50}$.

Aislamiento e identificación de fitoecdisteroides. Se aislaron e identificaron los fitoecdisteroides de la fracción más activa ( $n$-hexano). La fracción se sometió a cromatografía de columna en sílica gel (Merck, Kenilworth, N.J.; EE. UU.; Sigel grade $60,70-230,60 \mathrm{~A}^{\circ}$ ) usando un gradiente de polaridad desde $n$-hexano al $100 \%$ hasta acetato de etilo al $100 \%$. A partir de la polaridad intermedia ( $n$-hexano/acetato de etilo: 30/70) fue obtenida una mezcla rica en esteroles agrupada en una sola fracción, que fue sometida a una nueva cromatografía de columna. Los principales componentes obtenidos en ésta fueron purificados e identificados con base en evidencia espectroscópica (IR, RMN- ${ }^{1} \mathrm{H}$ y RMN $-{ }^{13} \mathrm{C}$ ). El espectro RMN $\left(250 \mathrm{MHz}\right.$ para ${ }^{1} \mathrm{H}$ y $65 \mathrm{MHz}$ para ${ }^{13} \mathrm{C}$ ) se midió en $\mathrm{CDCl}_{3}$ (que también proporcionó la señal de bloqueo) con espectrómetros Bruker (AC-250). El cambio químico ${ }^{13} \mathrm{C}$ fue asignado con la mejora sin distorsión por la polarización de transferencia (DEPT) usando un ángulo de giro de $135^{\circ}$. Un experimento NOESY fue realizado para determinar la estereoquímica del grupo -OH en C-6. El espectro IR fue registrado en un equipo Shimadzu FTIR-8400. Las rotaciones ópticas fueron medidas en cloroformo a $25^{\circ} \mathrm{C}$ (Alarcón et al. 2013). 


\section{Resultados}

Determinación de la incidencia de los extractos sobre las mortalidades en cada estadio y sobre la mortalidad acumulada en $T$. urticae cuando el tratamiento se realiza desde la etapa larval. La fracción hexánica, a una concentración de 100 ppm, (HB100) causó una mortalidad del $46,11 \%$ de los individuos en el estadio larval, la cual fue significativamente más alta que la registrada en los demás tratamientos. La fracción con acetato de etilo a 250 ppm (AB250) registró una tasa significativamente mayor de mortalidad de los individuos cuando se encontraban en el estadio ninfal $(64,39 \%)$ que los demás tratamientos, seguida de las fracciones hexánica a 250 ppm (HB250) y la obtenida con acetato de etilo a 100 ppm (AB100) $(54,61$ y $49,68 \%$, respectivamente) pero sin diferencias estadísticamente significativas entre estas dos últimas. Con respecto a los individuos que llegaron a la edad adulta, se registraron mortalidades significativamente más altas con los tratamientos realizados con las fracciones hexánica a 250 ppm (HB250), 100 ppm (HB100) y la obtenida con acetato de etilo a $10 \mathrm{ppm}$ (AB10). Al analizar la mortalidad acumulada se determinó que los tratamientos significativamente más efectivos, en cuanto a mortalidad, son HB250. HB100 y $\mathrm{AB} 100$, sin diferencias estadísticamente significativas entre ellos (Tabla 1).

Mortalidad de adultos. A las 24 y 48 horas de exposición se encontró que la fracción obtenida con acetato de etilo a 5.000 ppm (AB5000) presentaba diferencias estadísticamente significativas $(\mathrm{P} \leq 0,05)$ con respecto a los demás tratamientos. A las 72 horas después de la exposición AB5000 no presentó diferencias significativas con el testigo positivo (producto comercial) ni con los tratamientos realizados con la fracción hexánica a 2.500 y 5.000 ppm (HB2500 y HB5000 respectivamente). Estas dos últimas sí presentaron diferencias significativas con el resto de los tratamientos (Tabla 2).

En la Tabla 3 se pueden observar las $\mathrm{CL}_{50}$ de las dos fracciones para las pruebas de mortalidad acumulada cuando se sometió al ácaro desde su estadio larval y la de mortalidad cuando se aplicaron los tratamientos directamente sobre los adultos. Se puede observar que no hay diferencia significativa de las $\mathrm{CL}_{50}$ para las dos fracciones analizadas en todos los tiempos de observación dentro de cada prueba. Sin embargo, sí se encontraron diferencias significativas entre pruebas, mostrando claramente una mayor mortalidad
Tabla 2. Tasa de mortalidad (\%) de hembras adultas de Tetranychus urticae.

\begin{tabular}{cccc}
\hline \multirow{2}{*}{ Tratamiento } & \multicolumn{3}{c}{$\begin{array}{c}\text { Porcentaje de mortalidad en cada tiempo de } \\
\text { observación (horas) }\end{array}$} \\
\cline { 2 - 4 } & $\mathbf{2 4}$ & $\mathbf{4 8}$ & $\mathbf{7 2}$ \\
\hline HB5000 & $29,68( \pm 2,61) \mathrm{c}$ & $39,68( \pm 4,51) \mathrm{c}$ & $60,32( \pm 4,51) \mathrm{b}$ \\
HB2500 & $21,00( \pm 5,35) \mathrm{cd}$ & $25,76( \pm 4,65) \mathrm{cd}$ & $56,50( \pm 1,92) \mathrm{b}$ \\
HB1000 & $15,56( \pm 1,92) \mathrm{d}$ & $28,89( \pm 1,92) \mathrm{cd}$ & $35,56( \pm 1,92) \mathrm{c}$ \\
HB250 & $1,33( \pm 0,12) \mathrm{d}$ & $1,76( \pm 0,15) \mathrm{d}$ & $2,22( \pm 0,16) \mathrm{d}$ \\
$\mathrm{AB} 5000$ & $57,78( \pm 9,71) \mathrm{b}$ & $66,67( \pm 5,77) \mathrm{b}$ & $74,53( \pm 3,49) \mathrm{ab}$ \\
$\mathrm{AB} 2500$ & $8,89( \pm 3,85) \mathrm{d}$ & $13,33( \pm 5,77) \mathrm{d}$ & $22,22( \pm 1,92) \mathrm{cd}$ \\
$\mathrm{AB} 1000$ & $6,67( \pm 3,33) \mathrm{d}$ & $8,89( \pm 3,85) \mathrm{d}$ & $13,33( \pm 3,33) \mathrm{d}$ \\
AB250 & $0,00( \pm 0,00) \mathrm{d}$ & $0,00( \pm 0,00) \mathrm{d}$ & $0,00( \pm 0,00) \mathrm{d}$ \\
Testigo positivo & $100,00( \pm 0,00) \mathrm{a}$ & $100,00( \pm 0,00) \mathrm{a}$ & $100,00( \pm 0,00) \mathrm{a}$ \\
\hline
\end{tabular}

Las medias con la misma letra dentro de cada tiempo de observación (columna) no son diferentes significativamente según la prueba de Tukey (df $=49 ; \mathrm{P} \leq 0,05)(\mathrm{SAS}$ Institute 2004). La mortalidad fue corregida usando la fórmula de Abbott. Testigo negativo: agua destilada + tween 80 . Testigo positivo: Producto comercial con abamectina como ingrediente activo a 750 ppm.

cuando se somete al ácaro desde su estadio larval respecto al tratamiento que cuando se aplica directamente al adulto. No se obtuvo una $\mathrm{CL}_{50}$ significativa para los tiempos $24 \mathrm{y}$ 48 para la fracción hexánica en la prueba de mortalidad de adultos dado que no se presentaron mortalidades superiores al $50 \%$ con dichos tratamientos (Tabla 2).

Repelencia. Se observó actividad repelente significativa ( $\mathrm{P}$ $\leq 0,05)$ para los tratamientos realizados con las fracciones hexánicas a 10, 50 y 100 ppm $(66,20 \%$, 95,78\% y $100 \%$, respectivamente, a las 24 horas de observación) y con las obtenidas con acetato de etilo a $250 \mathrm{ppm}$, hasta las 4 horas de observación $(75,01 \%)$ y a 500 ppm, para todos los tiempos registrados $(95,75 \%$ a las 24 horas de observación), en comparación con los testigos negativo y positivo (producto comercial con extracto de Allium sativum como ingrediente activo). Todas las concentraciones usadas de la fracción hexánica y la fracción obtenida con acetato de etilo a 500 ppm (AB500) fueron significativamente superiores al testigo positivo en todos los tiempos de observación (Tabla 4).

Aislamiento e identificación de fitoecdisteroides. La fracción más activa en los tres tipos de bioensayos fue la obtenida a partir de $n$-hexano. A partir de ella se aislaron

Tabla 1. Porcentaje de mortalidad de individuos de Tetranychus urticae en cada estadio y mortalidad acumulada.

\begin{tabular}{|c|c|c|c|c|c|c|c|c|}
\hline Tratamiento & Larvas & $\begin{array}{c}\text { Intervalo de } \\
\text { confianza }\end{array}$ & Ninfas & $\begin{array}{c}\text { Intervalo de } \\
\text { confianza }\end{array}$ & Adultos & $\begin{array}{c}\text { Intervalo de } \\
\text { confianza }\end{array}$ & $\begin{array}{l}\text { Mortalidad } \\
\text { acumulada }\end{array}$ & Intervalo de confianza \\
\hline HB250 & $10,85 \mathrm{~b}$ & $(7,06-14,69)$ & $54,61 \mathrm{~b}$ & $(46,21-62,81)$ & $13,72 \mathrm{a}$ & $(8,42-19,02)$ & $79,18 \mathrm{a}$ & $(69,88-88,48)$ \\
\hline HB100 & $46,11 \mathrm{a}$ & $(42,32-49,90)$ & $18,04 \mathrm{~cd}$ & $(9,84-26,34)$ & $10,55 \mathrm{ab}$ & $(5,25-15,85)$ & $74,70 \mathrm{a}$ & $(65,40-84,00)$ \\
\hline HB10 & $10,00 \mathrm{bc}$ & $(6,21-13,79)$ & $23,82 \mathrm{c}$ & $(15,62-32,02)$ & $1,97 \mathrm{c}$ & $(-3,33-7,27))$ & $35,79 \mathrm{c}$ & $(26,48-45,08)$ \\
\hline $\mathrm{AB} 250$ & $6,75 \mathrm{c}$ & $(2,96-10,54)$ & $64,39 \mathrm{a}$ & $(56,19-72,59)$ & $8,41 \mathrm{~b}$ & $(3,11-13,71)$ & $79,55 \mathrm{a}$ & $(70,25-88,85)$ \\
\hline $\mathrm{AB} 100$ & $6,74 \mathrm{c}$ & $(2,95-10,53)$ & $49,68 \mathrm{~b}$ & $(41,48-57-88)$ & $0,20 \mathrm{c}$ & $(-5,10-5,50)$ & $56,62 \mathrm{~b}$ & $(47,32-65,92)$ \\
\hline $\mathrm{AB} 10$ & $6,30 \mathrm{c}$ & $(2,51-10,09)$ & $11,66 \mathrm{~d}$ & $(3,46-19,86)$ & $12,15 \mathrm{ab}$ & $(6,85-17,45)$ & $30,11 \mathrm{c}$ & $(20,82-39,42)$ \\
\hline
\end{tabular}

Las medias con la misma letra dentro de cada columna no presentan diferencias estadísticamente significativas según la prueba de Tukey $(\mathrm{df}=29 ; \mathrm{P} \leq 0,05)$ (StatPoint Technologies, Inc.2010). La mortalidad fue corregida usando la fórmula de Abbott. 
Tabla 3. Concentración letal media $\left(\mathrm{CL}_{50}\right)$ de las fracciones analizadas sobre hembras adultas de Tetranychus urticae.

\begin{tabular}{|c|c|c|c|c|c|c|c|c|}
\hline \multirow{3}{*}{$\begin{array}{l}\text { Tipo de } \\
\text { fracción }\end{array}$} & \multicolumn{8}{|c|}{$\mathbf{C L}_{50}(\mathbf{p p m})$ para las pruebas de mortalidad acumulada y mortalidad en hembras adultas } \\
\hline & \multicolumn{4}{|c|}{ Mortalidad acumulada } & \multicolumn{4}{|c|}{ Mortalidad en adultos } \\
\hline & 12 días & I.C. & 24 horas & I.C. & 48 horas & I.C & 72 horas & I.C. \\
\hline Hexano & 41,05 & $(-39,15-86,22) \mathrm{a}$ & N.S. & - & N.S. & - & 2816,72 & $(331,59-5340,92) b$ \\
\hline $\begin{array}{l}\text { Acetato de } \\
\text { etilo }\end{array}$ & 89,44 & $(49,47-127,18) \mathrm{a}$ & 4717,76 & $(4129,26-5640,84) b$ & 4256,97 & $(3723,04-5007,70) b$ & 3741,20 & $(3215,04-4430,41) b$ \\
\hline
\end{tabular}

Las $\mathrm{CL}_{50}$ obtenidas son significativas estadísticamente $(\mathrm{P} \leq 0,05$ en la tabla de análisis de desviaciones) dado que existe una relación estadísticamente significativa entre las variables concentración y mortalidad, con un nivel de confianza del 95,0\%. N.S.: No es significante (P $>0,05$ en la tabla de análisis de desviaciones) (StatPoint Technologies, Inc.,2010). Las $\mathrm{CL}_{50}$ con la misma letra no presentan diferencias estadísticamente significativas entre sí por el análisis de los intervalos de confianza (I.C.).

e identificaron los siguientes cuatro Fes: ecdisona, ponasterona, shidasterona y 2-deoxicrustecdisona (Fig. 1). A continuación, se expone la información detallada que permitió la elucidación de las estructuras.

Ecdisona (A). H RMN (C5D5N): $\delta 4,17$ (m, 1H, H-2), 4,21 (m, 1H, H-3), 3,01 (dd, 1H, H-5), 6,25 (d, 1H, H-7), 3,56 (m, 1H, H-9), 2,51 (s, 1H, H-17), 1,21 (s, 3H, H-18), 1,06 (s, 3H, H-19), 2,12 (s, 1H, H-20), 1,58 (s, 3H, H-21), 3,87 (m, 1H, H-22), 1,36 (s, 3H, H-26), 1,36 (s, 3H, H-27), C RMN(C5D5N): $\delta$ 38,08 (C-1), 68,10 (C-2), 68,10 (C-3), 32,45 (C-4), 51,41 (C-5), 203,36 (C-6), 121,61 (C-7), 165,53 (C-8), 34,63 (C-9), 38,76 (C-10), 21,20 (C-11), 31,49 (C-12), 47,70 (C-13), 83,97 (C-14), 32,03 (C-15), 26,74 (C-16), 48, 28 (C-17), 15,89 (C-18), 24,55 (C-19), 43,04 (C-20), 13,74 (C21), 74,07 (C-22), 25,69 (C-23), 42,55 (C-24), 68,80 (C-25), 30,09 (C-26), 30,01 (C-27).

Ponasterona A (B). HRMN (DMSO-d6) $\delta 3,60$ (m, 1H, H-2), 3,76 (s, 1H, H-3), 1,57 (m, 2H, H-4), 2,20 (dd, 1H, H-5), 8,51 (s, 1H, H-7), 3,0 (ddd, 1H, H-9), 2,23 (t, 1, H-17), 0,76 (s, $3 \mathrm{H}, \mathrm{H}-18$ ), 0,83 (s, 3H, H-19), 1,05 (s, 3H,H-21), 3,13 (dd, $1 \mathrm{H}, \mathrm{H}-22), 0,86(\mathrm{~s}, 3 \mathrm{H}, \mathrm{H}-26), 0,85$ (s, 3H, H-27), C RMN (DMSO-d6) $\delta 36,6$ (C-1), 66,8 (C-2), 66,6 (C-3), 31,6 (C-4), 50,1 (C-5), 202,7 (C-6), 120,4 (C-7), 165,2 (C-8), 33,2 (C-9), 37,6 (C-10), 20,3(C-11), 30,9(C-12), 46,9(C-13), 83,0(C-14), 30,3(C-15), 20,1(C-16), 48,7(C-17), 17,1(C-18), 23,9(C-19), 75,6(C-20), 20,9(C-21), 75,6(C-22), 29,1(C-23), 36,1(C-24), 27,5(C-25), 23,0(C-26), 22,3(C-27).
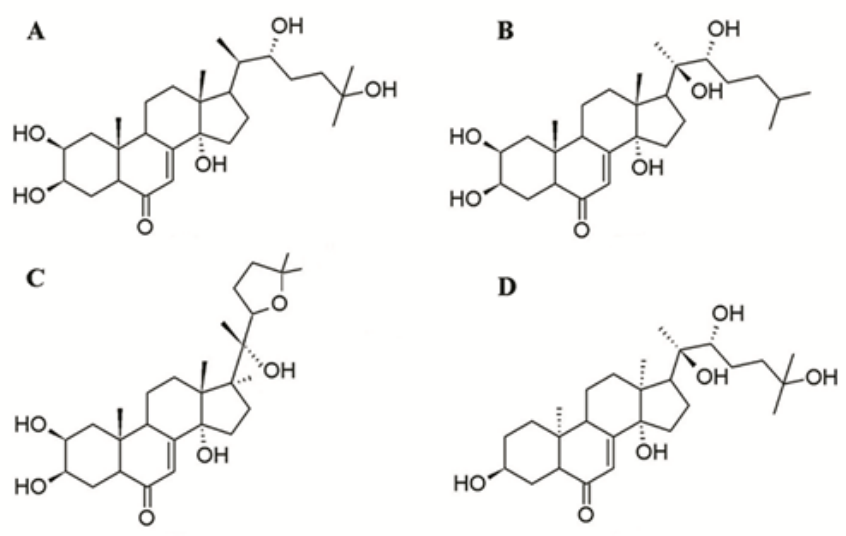

Figura 1. Fitoecdisteroides aislados de la fracción hexánica de Blechnum cordatum. A. Ecdisona. B. Ponasterona A. C. Shidasterona. D. 2-deoxicrustecdisona.
Shidasterona (C). H RMN (C5D5N): $\delta$ 4,10 (m, 1H, H-2), 4,19 (ddd, 1H, H-3), 2,95 (dd, 1H, H-5), 6,29 (d, 1H, H-7), 3,53 (ddd, 1H, H-9), 1,06 (s, 3H, H-18), 1,06 (s, 3H, H-19), 1,39 (s, 3H, H-21), 4,06 (dd, 1H, H-22), 1,22 (s, 6H, H-26,H-27), C RMN (C5D5N): $\delta 37,7$ (C-1), 67,9 (C-2), 67,9 (C-3), 32,2 (C-4), 51,2 (C-5), 203, 5(C-6), 121,5 (C-7), 166,0 (C-8), 34, 2 (C-9), 38,5(C-10), 21,0 (C-11), 31,5 (C-12), 47,7 (C-13), 84,0 (C-14), 31,5 (C-15), 21,5 (C-16), 51,2 (C-17)17,8 (C-18), 24,3 (C-19), 80,3 (C-20), 21,0 (C-21), 84,8 (C-22), 27,6 (C23), 38,8 (C-24), 75,4 (C-25), 28,2 (C-26), 28,7 (C-27), MS $\mathrm{m} / \mathrm{z} 485([\mathrm{M}+\mathrm{Na}]+13,7 \%), 463([\mathrm{M}+\mathrm{H}]+55 \%), 445$ $([\mathrm{M}+\mathrm{H}-\mathrm{H} 2 \mathrm{O}]+24 \%), 123(100 \%)$.

2-deoxicrustecdisona (D). H RMN (C5D5N): $\delta$ 3,83 (m, 1H, H-3), 2,29 (dd, 1H, H-5), 6,23 (d, 1H, H-7), 3,70 (ddd, 1H, H-9), 3,02 (t, 1H, H-17), 1,22 (s, 3H, H-18), 0,98 (s, 3H, H-19), 1,59 (s, 3H, H-21), 3,88 (brd, 1H, H-22), 1,37 (s, 3H, $\mathrm{H}-26), 1,37$ (s, 3H, H-27), C RMN(C5D5N): $\delta 31,71$ (C-1), 27,43 (C-2), 68,07 (C-3), 38,62 (C-4), 51,34 (C-5), 203,43(C6), 121,63 (C-7) , 166,02 (C-8), 34,46 (C-9), 37,95(C-10), 21,09 (C-11), 31,99(C-12), 48,09 (C-13), 84,18 (C-14), 32,37 (C-15), 21,40 (C-16), 50,08 (C-17), 17,84 (C-18), 24,41 (C19), 76,85 (C-20), 21,09 (C-21), 77,54 (C-22), 27,40 (C-23) 42,46 (C-24) , 69,56 (C-25), 29,96 (C-26) , 29,96 (C-27). MS $\mathrm{m} / \mathrm{z}$ (int.rel): 464 ([M], 1), 446 [M-H2O], 2), 347 (52), 329 (100), 285 (46), 234 (43), 147 (28), 121 (29), 99 (31).

De acuerdo con la inspección detallada de los datos que proporcionan los espectros $1 \mathrm{H}$ y $13 \mathrm{C}$ RMN para los compuestos A-D, permite observar en ellos señales características para compuestos del tipo fitoecdisteroides. En los compuestos A, C y D es posible apreciar un protón entre $6,25,6,29$ y $6,23 \mathrm{ppm}$ respectivamente, que es asignable al protón del sistema carbonilo $\alpha, \beta$-insaturado, que los fitoecdisteroides presentan en el anillo B. En el compuesto $\mathrm{B}$, producto del efecto solvente se observa mismo protón a $8,51 \mathrm{ppm}$. Lo anterior se confirma con la señal alrededor de 203 ppm correspondiente a un carbonilo la cual se asigna al C-6. Esta señal se acompaña de una insaturación de los carbones C-7 y C-8, observándose estos a 121 y 166 ppm respectivamente. El desplazamiento a campo bajo de C-22 (84,8 ppm) y C-25 (75.4) en el espectro C RMN del compuesto $\mathrm{C}$, permite postular la presencia de una función éter entre estos carbonos de la cadena lateral. La comparación de los datos espectroscópicos obtenidos con los reportados en la literatura confirma las estructuras reportadas.

No era objetivo del estudio realizar una identificación de todos los compuestos en la fracción ni los compuestos específicos responsables de las actividades encontradas. 
Tabla 4. Tasa de repelencia (\%) durante cada tiempo de observación.

\begin{tabular}{|c|c|c|c|c|c|}
\hline \multirow{2}{*}{ Tratamiento } & \multicolumn{5}{|c|}{ Horas después del tratamiento } \\
\hline & 1 & 2 & 3 & 4 & 24 \\
\hline HB100 & $100,00( \pm 0,00) \mathrm{a}$ & $100,00( \pm 0,00) \mathrm{a}$ & $100,00( \pm 0,00) \mathrm{a}$ & $100,00( \pm 0,00) \mathrm{a}$ & $100,00( \pm 0,00) \mathrm{a}$ \\
\hline HB50 & $93,75( \pm 2,89) \mathrm{a}$ & $93,75( \pm 2,89) \mathrm{a}$ & $94,24( \pm 2,89) \mathrm{a}$ & $100,00( \pm 0,00) \mathrm{a}$ & $95,78( \pm 2,89) \mathrm{a}$ \\
\hline HB10 & $56,23( \pm 5,77) b$ & $49,96( \pm 7,64) b$ & $65,40( \pm 5,00) \mathrm{a}$ & $80,01( \pm 7,64) \mathrm{a}$ & $66,20( \pm 7,64) b$ \\
\hline $\mathrm{AB} 500$ & $100,00( \pm 0,00) \mathrm{a}$ & $100,00( \pm 0,00) \mathrm{a}$ & $100,00( \pm 0,00) \mathrm{a}$ & $100,00( \pm 0,00) \mathrm{a}$ & $95,78( \pm 2,89) \mathrm{a}$ \\
\hline $\mathrm{AB} 250$ & $74,99( \pm 2,89) \mathrm{a}$ & $74,99( \pm 2,89) \mathrm{a}$ & $71,16( \pm 2,89) \mathrm{a}$ & $75,01( \pm 2,89) \mathrm{a}$ & $0,00( \pm 0,00) \mathrm{c}$ \\
\hline $\mathrm{AB} 100$ & $12,44( \pm 7,64) \mathrm{d}$ & $0,00( \pm 0,00) \mathrm{c}$ & $0,00( \pm 0,00) \mathrm{c}$ & $0,00( \pm 0,00) \mathrm{d}$ & $0,00( \pm 0,00) \mathrm{c}$ \\
\hline Testigo negativo & $0,00( \pm 0,00) d$ & $0,00( \pm 0,00) \mathrm{c}$ & $0,00( \pm 0,00) \mathrm{c}$ & $0,00( \pm 0,00) d$ & $0,00( \pm 0,00) \mathrm{c}$ \\
\hline Testigo positivo & $37,52( \pm 7,64) \mathrm{c}$ & $12,38( \pm 5,77) \mathrm{c}$ & $16,78( \pm 5,60) \mathrm{c}$ & $20,09( \pm 4,33) \mathrm{c}$ & $15,46( \pm 2,83) \mathrm{c}$ \\
\hline
\end{tabular}

Las medias con la misma letra dentro de cada tiempo de observación (columna) no son diferentes significativamente según la prueba de Tukey (df = 39; $\mathrm{P} \leq 0,05$ ) (SAS Institute 2004). Testigo negativo: agua destilada + tween 80. Testigo positivo: producto comercial con extracto de Allium sativum como ingrediente activo a $750 \mathrm{ppm}$.

\section{Discusión}

En el bioensayo donde se aplicaron los tratamientos a los discos de hoja, se ubicaron huevos de T. urticae y se registró la mortalidad de los ácaros desde el estadio de larva, la fracción hexánica de $B$. cordatum mostró ser la más efectiva, pues desde una concentración de 100 ppm se registró una reducción acumulada de la población del 74,7 \% (Tabla 1). En contraste, exponer a hembras adultas a las fracciones evaluadas requirió concentraciones significativamente superiores $(5.000 \mathrm{ppm})$ para obtener una tasa de mortalidad inferior $(60,32 \%)$ (Tabla 2) a las obtenidas con el experimento de mortalidad acumulada. La fracción hexánica a $250 \mathrm{ppm}$ causó una mortalidad acumulada, en el respectivo bioensayo, de 79,18 \% (Tabla 1) mientras que la misma concentración en el bioensayo de exposición directa a adultos solo generó una mortalidad de $2,22 \%$ a las 72 horas (Tabla 2). Los ensayos de repelencia mostraron que, aún a concentraciones muy bajas, se presentaron altas tasas de actividad repelente de ambas fracciones, especialmente la hexánica con 80,01 $\%$ a $10 \mathrm{ppm}$ (concentración varios órdenes de magnitud más baja que las usadas en el experimento de mortalidad de adultos), 4 horas después de la aplicación (Tabla 4). Estos resultados sugieren que la exposición temprana a dicha fracción debido, por lo menos en parte, a un posible efecto antialimentario de los compuestos presentes, evidenciado por la actividad repelente registrada, aún a concentraciones muy bajas. Las bajas tasas de mortalidad registradas cuando se exponen los adultos al tratamiento sugieren que, a pesar de existir indicios de toxicidad, no es esta actividad la principal responsable de la mortalidad acumulada registrada. No se encontraron investigaciones donde se hayan realizado este tipo de comparaciones en ácaros. Sin embargo, sí se encontraron algunos estudios realizados sobre insectos. Hincapié et al. (2011) encontraron que fracciones de la misma naturaleza de $B$. cordatum causaron pupación temprana y altas tasas de mortalidad acumulada en individuos de $D$. melanogaster expuestos a los tratamientos desde estadios larvales tempranos.

Algunos de los fitoecdisteroides reportados en este estudio han sido encontrados también en algunas especies de la familia Blechnaceae. Tal es el caso de la shidasterona (Takemoto et al. 1968) y ponesterona (Takemoto et al. 1969), ambas en B. niponicum; y ecdisona en B. minus (Chong et al. 1970) у B. vulcanicum (Russell et al. 1981).
Como se ha mencionado, hay ausencia de información con respecto a la actividad disuasoria de los FEs en ácaros. Sin embargo, los efectos disuasorios de algunos ecdisteroides en insectos son bien conocidos. En cuatro ínstares de $P$. interpunctella alimentados con una dieta que contenía cuatro ecdisteroides que también se encuentran en forma común en plantas (FEs), (20-E), ponasterona A (PonA), polipodina B (PolB), y makisterona A (MakA), se encontró que evadían el alimento dependiente de la concentración de dichos FEs, que fue desde 2 a $30 \mathrm{mM}$ (milimolar) (Rharrabe et al. 2011). En otro experimento, la ingestión de estos cuatro FEs a $200 \mathrm{ppm}$ sobre la misma especie de insecto causó disminución en el peso larval, indujo el canibalismo e incrementó la mortalidad, causada por una disrupción en el desarrollo (Rharrabe et al. 2010). Se ha encontrado que 20-E es un efectivo disuasor alimentario para larvas del primer ínstar de Spodoptera littoralis (Boisduval, 1833) (Lepidoptera: Noctuidae) y Ostrinia nubilalis (Hübner, 1796) (Lepidoptera: Crambidae) (Marion-Poll y Descoins 2002). Se comprobó que el sensillum gustatorio de larvas de Mamestra brassicae L., 1758 (Lepidoptera: Noctuidae) responde a bajas concentraciones de 20-E y PonA (Descoins y Marion-Poll 1999). Es importante recordar que PonA fue identificada también en este estudio como uno de los FEs presentes en $B$. cordatum.

Los ensayos de mortalidad en adultos mostraron que se necesitan altas concentraciones de las fracciones evaluadas para causar toxicidad directa sobre el ácaro adulto. Aly et al. (2011) encontraron que aplicando estándares comerciales de ecdisterona y $20-\mathrm{E}$ a $5 \mathrm{ppm}$ sobre $O$. perseae (Tetranychidae), se registraron tasas de mortalidad de $63,3 \%$ y $50 \%$, respectivamente. La diferencia en las concentraciones que causan efecto tóxico puede deberse a la baja concentración que tienen los FEs encontrados en las fracciones de $B$. cordatum, pero sería necesario cuantificar los mismos en dichos extractos, lo cual no fue uno objetivo de este estudio.

\section{Conclusiones}

Los extractos de B. cordatum, principalmente los de naturaleza apolar, constituyen una alternativa para el control de T. urticae, especialmente cuando su aplicación se hace desde el estadio larval. También, dichos extractos tienen un alto potencial para su uso como repelentes del ácaro. Los resultados obtenidos sugieren que los FEs identificados, 
dados sus antecedentes de actividad antialimentaria, juegan un papel importante en la actividad acaricida y en la repelencia registradas. El efecto repelente pudo no haber permitido la adecuada alimentación de los individuos, llevándolos a la muerte, cuando se aplicó el extracto desde el estadio larval. Para poder comprobar este planteamiento deben adelantarse, en el futuro, experimentos que permitan elucidar los mecanismos de acción de los ecdisteroides y FEs sobre T. urticae y otros ácaros. Estudiar el papel de los FEs sobre ácaros tetraníquidos abre la puerta para que puedan ser usados para su control.

\section{Agradecimientos}

Los autores agradecen a David Seigler (Plant Biology Dept. University of Illinois, en Urbana-Champaign, EE. UU.) por la identificación botánica de las muestras recolectadas; al Ministerio de Agricultura y Desarrollo Rural de Colombia, a la Universidad Pontificia Bolivariana, a la Universidad de Antioquia y Ceniflores (Colombia) y a la Dirección de Investigación de la Universidad del Bío-Bío (Grant DIUBB) (Chile).

\section{Literatura citada}

ABBOTT, W. S. 1925. A method of computing the effectiveness of an insecticide. Journal of Economic Entomology 18 (2): 265267. https://doi.org/10.1093/jee/18.2.265a

AFIFY, A. E. M. M.; ALI, F. S.; TURKY, A. 2012. Control of Tetranychus urticae Koch by extracts of three essential oils of chamomile, marjoram and eucalyptus. Asian Pacific Journal of Tropical Biomedicine 2 (1): 24-30. https://doi.org/10.1016/ S2221-1691(11)60184-6

ALARCÓN, J.; LAMILLA, C.; CÉSPEDES, C. L. 2013. Insecticidal activity of sesquiterpenes skeleton synthesized by the conventional Robinson annulations reaction on Drosophila melanogaster. Industrial Crops and Products 42: 268-272. https://doi.org/10.1016/j.indcrop.2012.05.026

ALY, R.; RAVID, U.; ABU-NASSAR, J.; BOTNICK, I.; LEBEDEV, G.; GAL, S.; ZIADNA, H.; ACHDARI, G.; SMIROV, E.; MEIR. A.; GHANIM, M. 2011. Biological activity of natural phytoecdysteroids from Ajuga iva against the sweetpotato whitefly Bemisia tabaci and the persea mite Oligonychus perseae. Pest Management Science 67 (12): 1493-1498. https:// doi.org/10.1002/ps.2203

ARAÚJO, M. J. C.; CÂMARA, C. A. G.; BORN, F. S.; MORAES, M. M.; BADJI, C. A. 2012. Acaricidal activity and repellency of essential oil from Piper aduncum and its components against Tetranychus urticae. Experimental \& Applied Acarology 57 (2): 139-155. https://doi.org/10.1007/s10493-012-9545-x

ARNAULT, C.; SLÁMA, K. 1986. Dietary effects of phytoecdysones in the leek-moth, Acrolepiopsis assectella Zell. (Lepidoptera: Acrolepiidae). Journal of Chemical Ecology 12 (10): 1979-1986. https://doi.org/10.1007/BF01041947

BATHORI, M.; TOTH, N.; HUNYADI, A.; MARKI, A.; ZADOR, E. 2008. Phytoecdysteroids and anabolic-androgenic steroids structure and effects on humans. Current Medicinal Chemistry 15 (1): 75-91. https://doi.org/10.2174/092986708783330674

BOYD, D. W.; ALVERSON, D. R. 2000. Repellency effects of garlic extracts on twospotted spider mite, Tetranychus urticae Koch. Journal of Entomological Science 35 (1): 86-90. https:// doi.org/10.18474/0749-8004-35.1.86

CABRERA, A. R.; DONOHUE, K. V; ROE, R. M. 2009. Regulation of female reproduction in mites: a unifying model for the Acari. Journal of Insect Physiology 55 (12): 1079-1090. https://doi. org/10.1016/j.jinsphys.2009.08.007

CHONG, Y. K.; GALBRAITH, M. N.; HORN, D. H. S. 1970. Isolation of deoxyecrustecdysone, deoxyecdysone, and $\alpha$-ecdysone from the fern Blechnum minus. Journal of the Chemical Society D 24: 1585-1692

DĄBROWSKI, Z. T.; SEREDYŃSKA, U. 2007. Characterization of the two-spotted spider mite (Tetranychus urticae Koch, Acari: Tetranychidae) response to aqueous extracts from selected plant species. Journal of Plant Protection Research 47 (2): 113-124.

DE-FU, C.; MING-XUE, S.; WEN-FU, X. 2002. Pesticidal character of phytoecdysteroids from Ajuga multiflora Bunge (Labiatae) on larvae of Cryptorrhynchus lapathi L. (Coleoptera: Curculionidae). Journal of Forestry Research 13 (3):177-182. https://doi.org/10.1007/BF02871693

DESCOINS, C. 2001. Perception de substances antiappétentes par des chenilles de lépidoptères phytophages. Anné Biologique 40: 55-73. https://doi.org/10.1016/S0003-5017(01)72085-5

DESCOINS, C.; MARION-POLL, F. 1999. Electrophysiological responses of gustatory sensilla of Mamestra brassicae (Lepidoptera, Noctuidae) larvae to three ecdysteroids: ecdysone, 20-hydroxyecdysone and ponasterone A. Journal of Insect Physiology 45 (10): 871-876. https://doi.org/10.1016/S00221910(99)00057-8

EDWARDS, C. A.; ARANCON, N. Q.; VASKO-BENNETT, M.; ASKAR, A.; KEENEY, G. 2010. Effect of aqueous extracts from vermicomposts on attacks by cucumber beetles (Acalymna vittatum) (Fabr.) on cucumbers and tobacco hornworm (Manduca sexta) (L.) on tomatoes. Pedobiologia 53 (2): 141-148. https:// doi.org/10.1016/j.pedobi.2009.08.002

GAUBE, F.; WÖLFL, S.; PUSCH, L.; WERNER, U.; KROLL, T. C.; SCHRENK, D.; HARTMANN, R. W.; HAMBURGER, M. 2008. Effects of Leuzea carthamoides on human breast adenocarcinoma MCF-7 cells determined by gene expression profiling and functional assays. Planta Medica 74 (14): 17011708. https://doi.org/10.1055/s-0028-1088316

GORELICK-FELDMAN, J.; MACLEAN, D.; ILIC, N.; POULEV, A.; LILA, M. A.; CHENG, D.; RASKIN, I. 2008. Phytoecdysteroids increase protein synthesis in skeletal muscle cells. Journal of Agricultural and Food Chemistry 56 (10): 35323537. https://doi.org/10.1021/jf073059z

HAMDEN, K.; AYADI, F.; JAMOUSSI, K.; MASMOUDI, H.; ELFEKI, A. 2008. Therapeutic effect of phytoecdysteroids rich extract from Ajuga iva on alloxan induced diabetic rats liver, kidney and pancreas. BioFactors 33 (3): 165-175. https://doi. org/10.1002/biof.5520330302

HINCAPIÉ, C. A.; LÓPEZ, G. E.; TORRES, R. 2008. Comparison and characterization of garlic (Allium sativum L.) bulbs extracts and their effect on mortality and repellency of Tetranychus urticae Koch (Acari: Tetranychidae). Chilean Journal of Agricultural Research 68 (4): 317-327. https://doi.org/10.4067/ S0718-58392008000400001

HINCAPIÉ, C. A.; MONSALVE, Z.; PARADA, K.; LAMILLA, C.; ALARCÓN, J.; CÉSPEDES, C. L.; SEIGLER, D. 2011. Insect growth regulatory activity of Blechnum chilense. Natural Product Communications 6 (8): 1085-1088. https://doi. org/10.1177/1934578X1100600808

HORIGANE, M.; OGIHARA, K.; NAKAJIMA, Y.; SHINODA, T.; TAYLOR, D. 2007. Cloning and expression of the ecdysteroid receptor during ecdysis and reproduction in females of the soft tick, Ornithodoros moubata (Acari: Argasidae). Insect Molecular Biology 16 (5): 601-612. https://doi.org/10.1111/ j.1365-2583.2007.00754.x

HORIGANE, M.; OGIHARA, K.; NAKAJIMA, Y.; TAYLOR, D. 2008. Isolation and expression of the retinoid $X$ receptor from last instar nymphs and adult females of the soft tick Ornithodoros moubata (Acari: Argasidae). General and Comparative Endocrinology 156 (2): 298-311. https://doi.org/10.1016/j. ygcen.2008.01.021

IDEAM. 2014. Cartas climatológicas - Medias mensuales (Medellín). Inf Histórica Clima Clim La Princ Ciudad 2014. Disponible en: http://bart.ideam.gov.co/cliciu/mede/temperatura.htm. [Fecha revisión: 15 noviembre 2014]. 
MALAUSA, T.; SALLES, M.; MARQUET, V.; GUILLEMAUD, T.; ALLA, S.; MARION-POLL, F.; LAPCHIN, L. 2006. Withinspecies variability of the response to 20-hydroxyecdysone in peach-potato aphid (Myzus persicae Sulzer). Journal of Insect Physiology 52 (5): 480-486. https://doi.org/10.1016/j. jinsphys.2006.01.007

MANNAN, M. M.; VICTOR, B.; MARIDASS, M. 2008. A review on the potential uses of ferns. Ethnobotanical Leaflets 12 (1): 281-285.

MARION-POLL, F.; DESCOINS, C. 2002. Taste detection of phytoecdysteroids in larvae of Bombyx mori, Spodoptera littoralis and Ostrinia nubilalis. Journal of Insect Physiology 48 (4): 467-476. https://doi.org/10.1016/S0022-1910(02)00068-9

MIGEON, A.; DORKELD, F. 2017. Spider mites web: a comprehensive database for the Tetranychidae. Tetranychus urticae. Disponible en: http://www1.montpellier.inra.fr/CBGP/ spmweb/notespecies.php?id=872. [Fecha revisión: 15 enero 2017]

MIRESMAILLI, S.; ISMAN, M. B. 2006. Efficacy and persistence of rosemary oil as an acaricide against twospotted spider mite (Acari: Tetranychidae) on greenhouse tomato. Journal of Economic Entomology 99 (6): 2015-2023. https://doi. org/10.1093/jee/99.6.2015

MUÑOZ, E.; LAMILLA, C.; MARIN, J. C.; ALARCON, J.; CESPEDES, C. L. 2013. Antifeedant, insect growth regulatory and insecticidal effects of Calceolaria talcana (Calceolariaceae) on Drosophila melanogaster and Spodoptera frugiperda. Industrial Crops and Products 42: 137-144. https://doi. org/10.1016/j.indcrop.2012.05.014

OGIHARA, K.; HORIGANE, M.; NAKAJIMA, Y.; MORIBAYASHI, A.; TAYLOR, D. 2007. Ecdysteroid hormone titer and its relationship to vitellogenesis in the soft tick, Ornithodoros moubata (Acari: Argasidae). General and Comparative Endocrinology 150 (3): 371-380. https://doi. org/10.1016/j.ygcen.2006.09.007

PAVELA, R.; STEPANYCHEVA, E.; SHCHENIKOVA, A.; CHERMENSKAYA， T.; PETROVA， M. 2016. Essential oils as prospective fumigants against Tetranychus urticae Koch. Industrial Crops and Products 94: 755-761. https://doi. org $/ 10.1016 /$ j.indcrop. 2016.09 .050

PRADA, C.; ROLLERI, C. H.; PASSARELLI, L. 2008. Morfología, caracterización y distribución geográfica de Blechnum cordatum (Blechnaceae-Pteridophyta). Acta Botanica Malacitana 33: 29-46.

RAMOS, J. P.; DE LA SOTA, E. R.; GIUDICE, G. E. 2006. Blechnum cordatum (Blechnaceae): Nueva cita para la flora del Noroeste de Argentina. Boletín de La Sociedad Argentina de Botánica 41 (1-2): 91-93.

RHARRABE, K.; SAYAN, F.; LAFONT, R. 2010. Dietary effects of four phytoecdysteroids on growth and development of the Indian meal moth, Plodia interpunctella. Journal of Insect Science 10 (13): 1-13. https://doi.org/10.1673/031.010.1301

RHARRABE, K.; SAYAH, F.; MARION-POLL, F. 2011. Gustatory perception of phytoecdysteroids in Plodia interpunctella larvae. Entomologia Experimentalis et Applicata 138 (1): 33-39. https:// doi.org/10.1111/j.1570-7458.2010.01068.x

RIDDIFORD, L. M. 2012. How does juvenile hormone control insect metamorphosis and reproduction? General and Comparative Endocrinology 179 (3): 477-484. https://doi.org/10.1016/j. ygcen.2012.06.001

RUSSELL, G. B.; GREENWOOD, D. R.; LANE, G. A.; BLUNT, J. W.; MUNRO, M. H. G. 1981. 2-deoxy-3-epiecdysone from the fern Blechnum vulcanicum. Phytochemistry 20: 2407-2410. https://doi.org/10.1016/S0031-9422(00)82676-X

SAS INSTITUTE INC. 1999. SAS OnlineDoc $\AA$, version 8, Cary, NC, SAS Institute Inc. EE. UU.

SEIXAS, A.; FRIESEN, K. J.; KAUFMAN, W. R. 2008. Effect of 20-hydroxyecdysone and haemolymph on oogenesis in the ixodid tick Amblyomma hebraeum. Journal of Insect Physiology 54 (7): 1175-1183. https://doi.org/10.1016/j.jinsphys.2008.05.004

SIVIRA, A.; SANABRIA, M.; VALERA, N.; VÁSQUEZ, C. 2011. Toxicity of ethanolic extracts from Lippia origanoides and Gliricidia sepium to Tetranychus cinnabarinus (Boisduval) (Acari: Tetranychidae). Neotropical Entomology 40 (3): 375379.

STATGRAPHICS TECHNOLOGIES, INC. 2013. Statgraphics Centurion XVI. The Plains, VA, EE. UU.

TAKEMOTO, T.; HIKINO, Y.; OKUYAMA, T.; ARIHARA, S.; HIKINO, H. 1968. Structure of shidasterone, a novel insectmoulting substance from Blechnum niponicum. Tetrahedron Letters 9: 6095-6098. https://doi.org/10.1016/S00404039(00)70803-3

TAKEMOTO, T.; OKUYAMA, T.; ARIHARA, S.; HIKINO, Y.; HIKINO, H. 1969. Isolation of insect-moulting substances from Blechnum amabile and Blechnum niponicum. Chemical \& Pharmaceutical Bulletin 17: 1973-1974. https://doi.org/10.1248/ cpb.17.1973

VAN LEEUWEN, T.; VONTAS, J.; TSAGKARAKOU, A.; DERMAUW, W.; TIRRY, L. 2010. Acaricide resistance mechanisms in the two-spotted spider mite Tetranychus urticae and other important Acari: a review. Insect Biochemistry and Molecular Biology 40 (8): 563-72. https://doi.org/10.1016/j. ibmb.2010.05.008

ZAPATA, N.; CEBALlOS, R.; CÉSPEDES, C.; ALARCON, J.; LEYTON, A. 2016. Actividad insecticida y reguladora del crecimiento de extractos de Blechnum chilense (Blechnaceae) y Condalia microphylla Cav. (Rhamnaceae), sobre larvas de Galleria mellonella (L.) (Lepidoptera: Pyralidae). Boletín Latinoamericano y del Caribe de Plantas Medicinales y Aromáticas 15 (2): 77-87.

\section{Origen y financiación}

La investigación que originó el artículo hizo parte de la tesis doctoral de Carlos Augusto Hincapié Llanos "Evaluación de la actividad acaricida de extractos de plantas sobre Tetranychus urticae Koch y Amblyseius californicus McGregor" en el doctorado en Biología de la Universidad de Antioquia en 2012. Parte de la investigación se llevó a cabo durante la pasantía doctoral realizada en la Universidad del Bio Bio en la ciudad de Chillán en Chile. La financiación de la investigación donde se enmarcó la tesis mencionada fue realizada por el Ministerio de Agricultura y Desarrollo Rural de Colombia.

\section{Contribución de los autores}

Carlos A. Hincapié: realizó la búsqueda bibliográfica, la cría del ácaro, las extracciones, los experimentos, el análisis de estos y la escritura de la mayor parte del artículo.

Zulma Isabel Monsalve: dirigió la tesis doctoral, revisó los experimentos y sus análisis, revisó y corrigió todos los documentos que se generaron durante esta investigación.

Julio Alarcón: coordinó la obtención del material vegetal de B., y la identificación de los compuestos presentes en los extractos. También, aportó información para la escritura del artículo y revisó cada una de las versiones de este.

Carlos Céspedes: coordinó todas las extracciones, el diseño de los experimentos con los ácaros, aportó información para la escritura del artículo y revisó las versiones de este.

La planta con que se trabajó hacia parte de una investigación que estaban realizando los dos últimos coautores. Ellos además coordinaron todo el trabajo realizado durante la pasantía en la Universidad del Bio Bio en Chile. 